\title{
Discrete Random Contention System with Variable Packet Length Based on Binary Tree Conflict Resolution Algorithm
}

\author{
Jing Nan \\ School of Information, Yunnan University \\ Kunming, Yunnan, China \\ E-mail: 381239578@qq.com \\ Hongwei Ding \\ School of Information, Yunnan University \\ Kunming, Yunnan, China \\ E-mail: dhw1964@163.com
}

\author{
Yingying Guo \\ School of Information, Yunnan University \\ Kunming, Yunnan, China \\ E-mail: 674987178@qq.com \\ Qianlin Liu \\ Military Command Region of Yunnan Province \\ Kunming, Yunnan, China \\ E-mail: liuqianlin@sina.com
}

\begin{abstract}
The paper researches the random contention system based on the binary tree conflict resolution algorithm to improve the throughput in-depth using the average cycle method, then gets the formulas of the systemic throughput and so on. The simulation results verify the correctness of the theory, meanwhile, gets some conclusions that the different arrival rate $G$ is how to affect the main source of the throughput with variable packet length. It has some researching significance and improves the system performance.
\end{abstract}

Keywords- the average cycle method; improved binary tree conflict resolution algorithm; throughput; discrete random contention system; arrival rate

\section{INTRODUCTION}

With the development of computer technology, network technology and wireless communication technology, many the information retrieval modes and processing modes appear in the electronic world with smaller, cheaper and low-power system added network technology, wireless sensor network ${ }^{[1]}$ is an area which has a representative.

Wireless sensor network has a strong correlation of applications. That is, for different applications, need to adopt different strategies, in order to achieve the purpose that the developed wireless sensor network can fit the applications well. With the progress of the times, the applications of wireless sensor network in every aspect will be increased, and some of the existing protocol cannot meet these requirements well, so need to introduce some of the new protocols ${ }^{[2]}$.

This paper mainly introduced a discrete random contention system based on improved binary tree conflict resolution algorithm. The successful packet length is variable. Simulation results verify the correctness of the theory analysis ${ }^{[3]}$, and the performance of system has been greatly improved when introduce the improved binary tree conflict resolution algorithm. The inadequacy of the system is to increase the complexity.

\section{THE ANALYSIS OF DISCRETE RANDOM SYSTEM}

Assume that the discrete random contention systemic timeline is divided into equal length, and the length of each time slot is $\mathrm{L}$ on the channel ${ }^{[4]}$. With no limit on the number of system terminals, the packet signal arriving at any time will be transmitted in the beginning of next slot ${ }^{[5]}$. Therefore, there will be three interleaved random kinds of events which occurred in the discrete random contention system, (1) the packet is sent successfully (U); (2) the packet conflict (B); (3) idle timeslot (I).

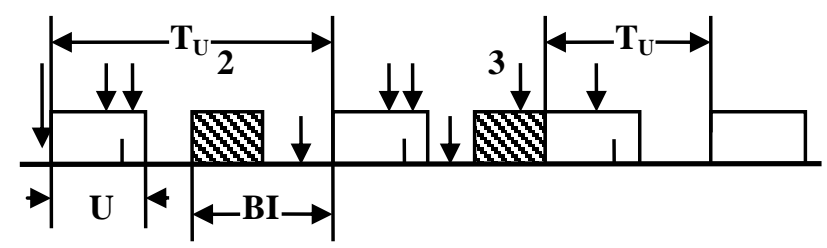

Figure 1. The random event graph of random contention system

As shown in the Fig.1, the collision event and idle event composite to be seen as a kind of event ${ }^{[6]}$, then events occurring on the time axis are reduced into two events, that is the event that the packet was sent successfully (U) and the composite event (BI), and these events on the timeline is the endless cycle occurs, the cyclical time variable is said $\mathrm{Tu}$.

The assumption is shown as follows before analyze the system performance:

1. The information packets arrival on the channel follows the Poisson distribution with an independent parameter $\mathrm{G}$, where $\mathrm{G}$ is arrival rate.

2. The channel is ideal; the packet on the channel is not affected by any noise and interference.

3. Assume that the channel on the time axis is divided into slot of equal length, and each slot length is L.

4. Packets arrived during the channel is busy to transmit information are remitted to the beginning of next timeslot to transmit. 
5. Assume packets arrived during idle time or collision time collides on the channel, then the impacted packets stop sending information at end of the timeslot.

The probability of the packet is transmitted successfully within a time slot is ${ }^{[7]}$ :

$$
P_{U}=P(1)=\frac{(G t)^{k} e^{-G t}}{k}=G L e^{-G L}
$$

The probability of no packet on the channel is:

$$
P_{I}=P(0)=\frac{(G t)^{k} e^{-G t}}{k}=e^{-G L}
$$

The joint probability that appears i successful events and $\mathrm{j}$ composite events continuously in a cycle period is:

$$
P(i, j)=\left(G L e^{-G L}\right)^{i}\left(1-G L e^{-G L}\right)^{j}
$$

The average number of the successful event is:

$$
E(i)=\sum_{i=1}^{\infty} \sum_{j=1}^{\infty} i P(i, j)=\frac{1}{1-G L e^{-G L}}
$$

The average number of the composite event is:

$E(j)=\sum_{i=1}^{\infty} \sum_{j=1}^{\infty} j P(i, j)=\frac{1}{G L e^{-G L}}$

The mean length of the successful event is:

$$
E(U)=E(i) L_{U}=\frac{L_{U}}{1-G L e^{-G L}}
$$

The probability that appear $\mathrm{k}$ composite events is:

$$
\begin{aligned}
& P(B I=k)=\left(1-G L e^{-G L}\right)^{k} \\
& =\sum_{l=0}^{k} C_{k}^{l}\left(e^{-G L}\right)^{l}\left(1-e^{-G L}-G L e^{-G L}\right)^{k-l}
\end{aligned}
$$

Then the average number of idle event is:

$$
\begin{aligned}
& E\left(N_{I}\right)=\sum_{i=1}^{\infty}\left(G L e^{-G L}\right)^{i} \sum_{k=1}^{\infty} \sum_{l=0}^{k} l C_{k}^{l}\left(e^{-G L}\right)^{l}\left(1-e^{-G L}-G L e^{-G L}\right)^{k-l} \\
& =\frac{1}{G L\left(1-G L e^{-G L}\right)}
\end{aligned}
$$

The mean length of the composite event BI in a cycle period is:

$$
\begin{aligned}
& E(B I)=E\left(N_{B}\right) L_{B}+E\left(N_{I}\right) L_{I} \\
& =\frac{L_{B}\left(1-e^{-G L}-G L e^{-G L}\right)+L_{I} e^{-G L}}{G L e^{-G L}\left(1-G L e^{-G L}\right)}
\end{aligned}
$$

The average of $\mathrm{Tu}$ is:

$$
\begin{aligned}
& E\left(T_{U}\right)=E(U)+E(B I) \\
& =\frac{L_{U} G L e^{-G L}+L_{B}\left(1-e^{-G L}-G L e^{-G L}\right)+L_{I} e^{-G L}}{G L e^{-G L}\left(1-G L e^{-G L}\right)}
\end{aligned}
$$

The systemic throughput is:

$$
\begin{aligned}
& S_{U}=\frac{E(U)}{E\left(T_{U}\right)} \\
& =\frac{L_{U} G L e^{-G L}}{L_{U} G L e^{-G L}+L_{B}\left(1-e^{-G L}-G L e^{-G L}\right)+L_{I} e^{-G L}}
\end{aligned}
$$

The average number of slot in a cycle time by the joint probability is ${ }^{[8]}$

$$
E\left(N_{I}\right)=\frac{P_{0}}{P_{1}\left(1-P_{1}\right)}=\frac{e^{-G L}}{G L e^{-G L}\left(1-G L e^{-G L}\right)}
$$

There are $x(x \geq 2)$ information packets impacted in a slot, then the average number slots that there are $x$ packets conflict in a cycle time is

$$
\begin{aligned}
& E\left(N_{B x}\right)=\frac{P_{x}}{P_{1}\left(1-P_{1}\right)} \\
& =\frac{(G L)^{x} e^{-G L}}{x ! G L e^{-G L}\left(1-G L e^{-G L}\right)}
\end{aligned}
$$

The average length of the idle time in a cycle time is

$$
E(I)=\frac{e^{-G L}}{G L e^{-G L}\left(1-G L e^{-G L}\right)}
$$

The average length of successful packets in a cycle time is

$$
E(U)=E\left(U^{*}\right)=\frac{L_{U}}{1-G L e^{-G L}}
$$

The average length of that there are $x$ information packets impacted in a cycle time is ${ }^{[9]}$

$$
\begin{aligned}
& E\left(B_{x}\right)=E\left(N_{B x}\right) x \frac{(G L)^{x} e^{-G L}}{(x-1) ! G L e^{-G L}\left(1-G L e^{-G L}\right)} \\
& E\left(B_{x}^{*}\right)=E\left(N_{B x}\right)\left(1+\overline{L_{x}}\right) \\
& =\frac{(G L)^{x} e^{-G L}\left(1+\overline{L_{x}}\right)}{x ! G L e^{-G L}\left(1-G L e^{-G L}\right)}
\end{aligned}
$$

From the definition of the system throughput we can get the throughput of the discrete random system based on the improved binary tree conflict resolution algorithm

$$
S=\frac{\left(L_{u}+e^{G L}-1\right) G L}{1+L_{u} G L+e^{G L}-G L+\sum_{x=2}^{\infty} \frac{\bar{L}_{x}(G L)^{x}}{x !}}
$$

$\overline{L_{x}}$ is the system average slot length when using the improved binary tree conflict resolution algorithm ${ }^{[10]}$. 


\section{THEORETICAL CALCULATION AND SIMULATION EXPERIMENT}

On the basis of the above analysis, we conducted computer simulations on the discrete random contention system, the experimental parameters are the same with the theoretical parameters, the length of the slot and packet are both unit length. The results are shown in the Table1.

TABLE.1 THE COMPARISON OF EXPERIMENT VALUE AND THEORETICAL VALUE

\begin{tabular}{ccccccc}
\hline & \multicolumn{2}{c}{ Su } & \multicolumn{2}{c}{ Eu } & \multicolumn{2}{c}{ E (Tu) } \\
& experiment value & theoretical value & experiment value & theoretical value & experiment value & theoretical value \\
\hline 0.1 & 0.090495 & 0.090484 & 1.0994 & 1.0995 & 8.229800 & 8.229600 \\
0.3 & 0.222260 & 0.222250 & 1.2859 & 1.2858 & 1.920700 & 1.920600 \\
0.5 & 0.303270 & 0.303270 & 1.4351 & 1.4353 & 0.845180 & 0.845180 \\
0.7 & 0.347570 & 0.347610 & 1.5328 & 1.5328 & 0.462760 & 0.462810 \\
0.9 & 0.365880 & 0.365910 & 1.5769 & 1.5771 & 0.286420 & 0.286450 \\
1.1 & 0.366120 & 0.366160 & 1.5775 & 1.5777 & 0.191790 & 0.191810 \\
1.3 & 0.354260 & 0.354290 & 1.5485 & 1.5487 & 0.135350 & 0.135370 \\
1.5 & 0.334690 & 0.334700 & 1.5029 & 1.5031 & 0.098956 & 0.098966 \\
1.7 & 0.310530 & 0.310560 & 1.4506 & 1.4505 & 0.074088 & 0.074088 \\
1.9 & 0.284150 & 0.284180 & 1.3969 & 1.3970 & 0.056354 & 0.056350 \\
\hline
\end{tabular}

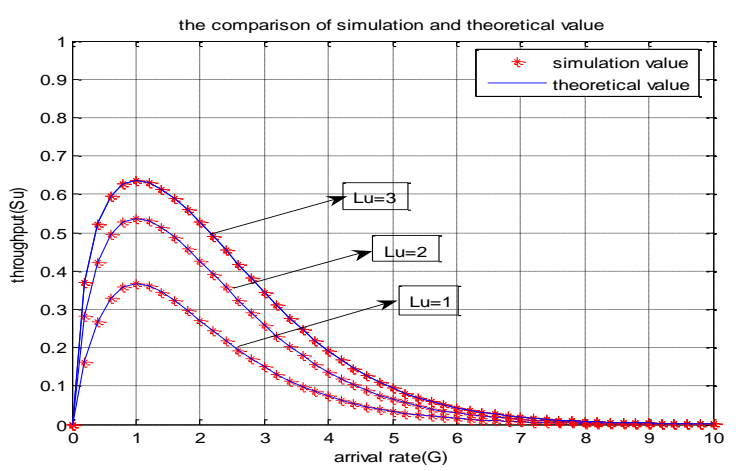

Figure 2. The changing curve of the throughput with different $\mathrm{Lu}$

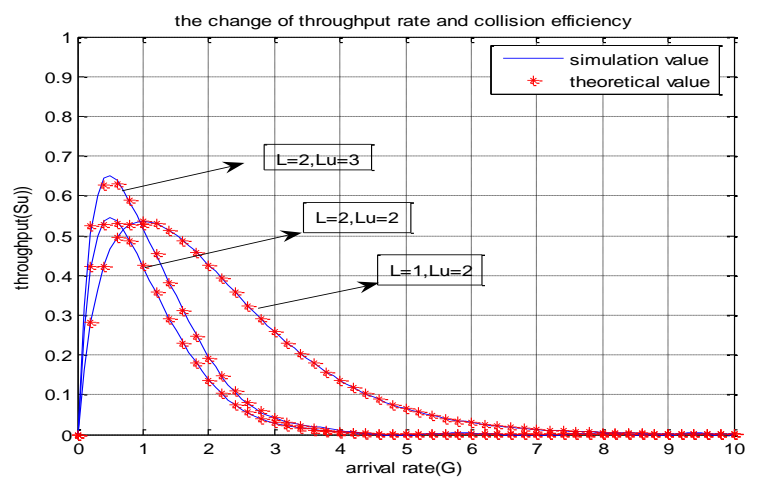

Figure 3. The changing curve of the throughput with different $\mathrm{L}$

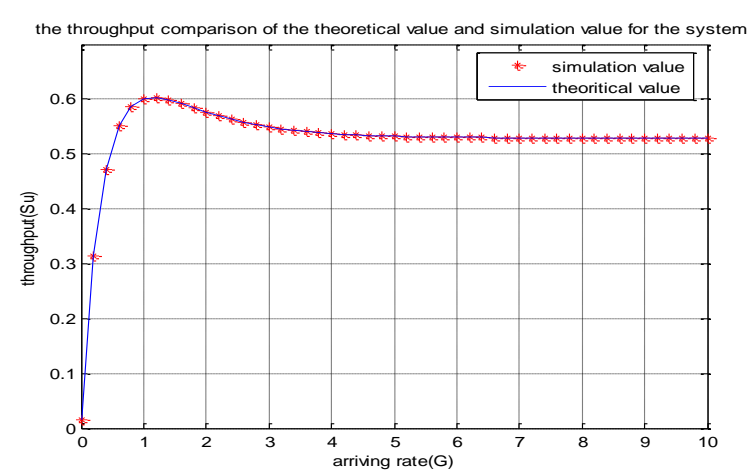

Figure 4. The throughput comparison of theory and simulation

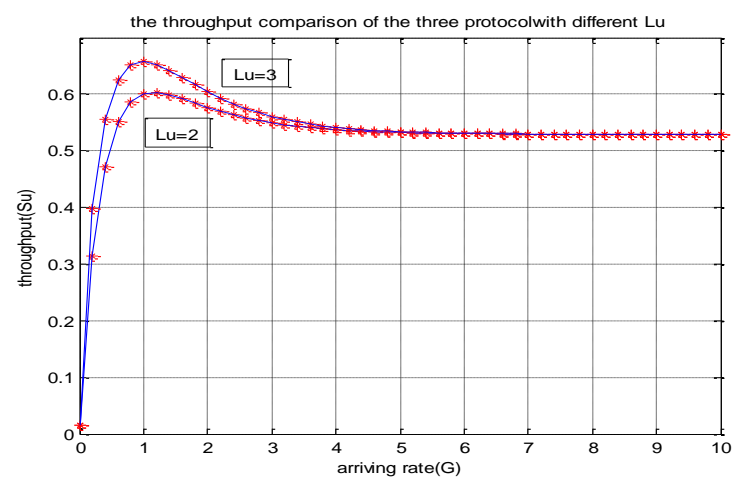

Figure 5. The throughput comparison of the protocol with different $\mathrm{Lu}$ 


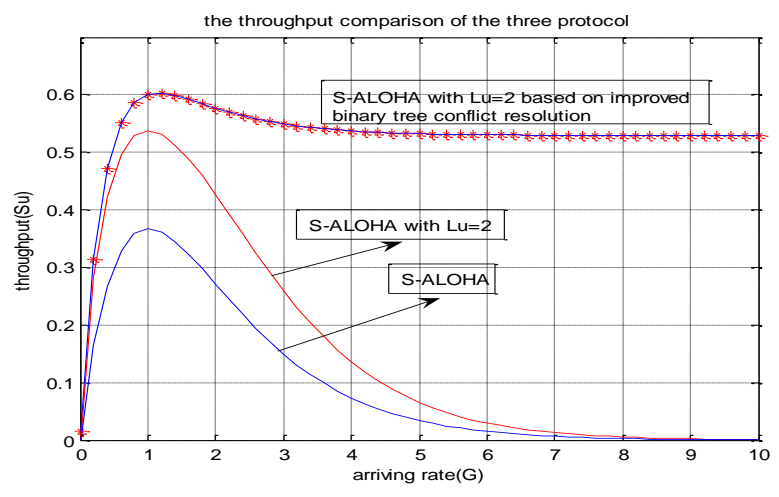

Figure 6. The comparison of the three protocol

From above tables and graphs, we can receive:

(1) With the increases of the successful packet length $\mathrm{Lu}$, the maximum throughput of the system also increases, and reached the maximum in the arrival rate $G=1$. For example, when $\mathrm{Lu}=1$, the maximum throughput $\mathrm{S}$ is 0.368 , and when $\mathrm{Lu}=2, \quad \mathrm{Smax}=0.537$, and when $\mathrm{Lu}=3$, Smax $=0.626$, the system throughput is obvious has been improved.

(2) The slot length $L$ does not affect the value of the maximum throughput, it just change the value of $G$ reaches the maximum throughput. When the $\mathrm{L}$ is increased, the system will be achieving maximum throughput in smaller G.

(3) The discrete random contention system based on the improved binary tree conflict resolution algorithm improved the throughput than the discrete random contention system with $\mathrm{Lu}=2$ and traditional slotted Aloha.

(4) Whether traditional slotted Aloha protocol, or the discrete random contention with $\mathrm{Lu}=2$, the throughput approaches to zeros when having a heavy load. And the throughput of the discrete random contention system based on the improved binary tree conflict resolution algorithm has been maintained at about 0.529 when the load increases.

(5) The theoretical value and simulation value of the throughput for the discrete random contention system based on the improved binary tree conflict resolution algorithm coincide with each other to prove the correctness of the theory analysis. And when the length of successful packet increases, the maxmium throughput will increases also.

\section{CONCLUSION}

This paper mainly introduced a discrete random contention system based on improved binary tree conflict resolution algorithm. By building mathematical model and using, mathemat ical methods to analyze and simulate the new random discrete random access protocol based on improved binary tree conflict resolution algorithm, and analyze performance of the throughput that reflected on the curves. Simulation results verify the correctness of the theory, we know that the performance of system has been greatly improved when introduce the improved binary tree conflict resolution algorithm. The inadequacy of the system is to increase the complexity.

\section{CORRESPONDING AUTHOR}

Hongwei Ding (1964-), male, Professor of Yunnan University, PhD Degree. Mainly engaged in the research of random multiple access communication system, polling system, network communication engineering.

\section{ACKNOWLEDGMENT}

This work was supported by the National Natural Science Foundation of China (61072079); Natural Science Foundation of Yunnan Province (2010CD023); Graduate Scientific Research Fund of Yunnan University (ynuy201047).Thanks to the seriously guidance of Professor Dongfeng Zhao.

\section{REFERENCES}

[1] Qin Danyang, Wang Huiwu, Ma Lin, Ding Qun. Research on Topology Property for Wireless Multihop Communication Network. TELKOMNIKA Indonesian Journal of Electrical Engineering. 2013, 1(11): 351-361.

[2] Sallent, O.; Agusti, R.A proposal for an adaptive S-ALOHA access system for a mobile CDMA environment, Vehicular Technology, IEEE Transactions on Industrial, 1998.977- 986.

[3] A. El-Hoiydi. ALOHA with preamble sampling for sporadic traffic in ad hoc wireless sensor networks. IEEE International Conference on Communications (ICC), New York, Apr. 2002.

[4] Hermawan Hermawan, Indonesia; Riyanarto Sarno. Developing Distributed System With Service Resource Oriented Architecture. TELKOMNIKA Indonesian Journal of Electrical Engineering. 2012, 2(10): 389-399.

[5] Zhao Dongfeng, Li Bihai, Zheng Sumin, Sun Quan. Study of Mean Cycle Times for Slotted ALOHA System. Journal of Yunnan University (Natural Science), 1994, 16(2): 133- 138.

[6] Y.-W. P. Hong, C.-K. Lin, and S.-H. Wang. Exploiting Cooperative Advantages in Slotted

[7] ALOHA Random Access Networks. IEEE Trans. on Inform. Theory, 2010, IT-56(8): 3828-3846.

[8] Zhao Dongfeng, Li Bihai, Zheng Sumin. Study on a New Method for the Slotted Access Channel. Journal of Electronic Science. 1997, 19(6): 814- 819

[9] Zhao Dongfeng. Study on the Average Cycle Method for Slotted Multiple Access Communications. Journal of China Institute of Communications. 1999, 20(8):80-85.

[10] Hou Jianbin. Study on S-ALOHA based on Unfenced Two-ray Tree Algorithm of Packet Conflict Resolution. Yunnan University, 2011.

[11] Xu Bo. Analysis on 1-persistent CSMA protocol based on Tree Algorithm of Packet Conflict Resolution. Yunnan University, 2011.

[12] Gao Yangshui. 1-insist CSMA and Tree Conflict Decomposition Algorithm Convergence Protocol and Application circuit Design. Yunnan University, 2012.

[13] Yingtu W, Jinfu C1Collision resolution for variable 2 length messages1IEEE Trans-Communication 1993, COM 241 (9): 12811283.

[14] Zhao H,Wang S,Xi Y,et al. Modeling intra-flow contention problem in wireless multi-hop networks .IEEE Commun Lett, 2010, (14) : 18-20. 